\title{
Patient safety and nutrition: is there a connection?
}

\author{
Christina Nieuwoudt, Registered Dietitian; Part-Time Lecturer, University of Pretoria \\ Past Chairperson, Al Rahba Hospital Patient Safety Committee, Abu Dhabi, United Arab Emirates \\ Correspondence to: E-mail: foodfundi@yahoo.co.uk \\ Keywords: dietetic students, Three-Factor Eating Questionnaire, Eating Attitudes Test 26, body mass index, eating behaviour, eating disorders
}

\begin{abstract}
Nutrition care is not always recognised as a patient safety issue. This article explores the origins of the patient safety initiative and seeks to identify possible connections between nutrition care and patient safety. Examples of tools that can be used to improve the safety of nutrition care are provided. This is also a call to action as patient safety data for nutrition care in the South African context are lacking and much can be learned from worldwide patient safety initiatives.
\end{abstract}

(P) Peer reviewed. (Submitted: 2013-09-30. Accepted: 2013-12-07) ๑ SAJCN

S Afr J Clin Nutr 2014;27(3):102-105

\section{Introduction}

To understand a possible connection between patient safety and nutrition, the origins of the patient safety initiative need to be explored. It has its origins in 1966, when Avedis Donabedian, a professor at Harvard University, understood health care to be a system. He dedicated the rest of his professional life to the theoretical advancement of quality improvement and health services research. ${ }^{1}$ The medical community "discovered" patient safety with the publication of a report by the Institute of Medicine in the USA called To err is human: building a safer healthcare system in $1999 .{ }^{2}$ This report states that between 44000 and 98000 people die in USA hospitals each year because of preventable medical errors. Before the report was published, there was sporadic interest in patient safety, e.g. only occurring when the media covered a sensational medical error. Examples of such cases included the death of a celebrity owing to a chemotherapy overdose, and another who had the "wrong" leg amputated. ${ }^{3}$ In the report, "patient safety" is defined as freedom from accidental injury. "Error" is defined as the failure of a planned action to be completed as intended, or the use of an incorrect plan to achieve an aim. Errors can occur at all stages in the process of care, from diagnosis to treatment to preventive care. To prevent errors, healthcare systems must be designed that are safer at all levels. Blaming individuals is counterproductive. Safety should be built into healthcare processes. This is considerably more effective than blaming an individual. To err is human, but errors can be prevented. Therefore, errors should be analysed so that lessons can be learnt from the mistakes made and processes established to prevent errors.

Another report, Crossing the quality chasm: a new health system for the $21^{\text {st }}$ century, published by the Institute of Medicine in 2001 recognised that there was a large chasm between the quality of the health care that should be provided and what was provided. It was clear that expectations were not being met. Six areas of improvement were identified with regard to being able to cross the quality chasm. The first was safe health care for patients. ${ }^{4}$

As one in 10 patients around the world is affected by medical errors, in 2005, the World Health Organization (WHO) designated the Joint Commission and Joint Commission International as the WHO collaborating centre for patient safety solutions. Recognised as a leader in patient safety, the Joint Commission promotes the delivery of safe, high-quality care through its standards; sentinel event database, Sentinel Event Alert; the prevention of patient care handover errors; Speak Up ${ }^{\mathrm{TM}}$ programme and national patient safety goals. The collaboration centre then identified the nine most common medical errors and developed solutions to them. These are available to WHO members to help them to improve patient safety within the healthcare systems. ${ }^{5}$ Of the solutions, the following link to nutrition: patient identification, assuring medication accuracy during transitions in care, and avoiding catheter and tubing misconnections.

\section{Nutrition support and patient safety}

Safe therapy is a first priority in patients requiring nutrition support therapy. ${ }^{6}$ However, this was not always the case. The National Patient Safety Agency in the UK maintains a register of all patient safety incidents reported in the UK through the National Reporting and Learning System. In a report published in 2010, 1433 incidents met the definition of nutrition-related incidents. An "incident" was defined as a nutrition-related patient safety incident that either caused harm or had the potential to cause harm to an individual. Twenty-three per cent of these nutrition-related incidents were in the category "Provision of nutrition via artificial feeding". ${ }^{7}$ Furthermore, as 
recently as 2011, The Institute for Safe Medication Practices Canada reported tubing misconnections that caused premature infants to receive breast milk intravenously, this despite the industry's efforts to prevent these events by producing incompatible intravenous and enteral tubing connections. ${ }^{8}$

\section{Healthcare practices with the potential to cause nutrition-related patient safety incidents}

\section{Patient identification}

Failure to correctly identify patients continues to result in medical errors. It has been recommended that two identifiers should be used to identify a patient and that bed or room numbers should not be used. It is quite common that healthcare professionals use a bed or room number to identify a patient, but this practice lends itself to errors as the patient might be moved to another location, or patients with the same surname could be mixed up. ${ }^{9}$ Therefore, it is possible that a patient might not receive the prescribed nutrition support. Failure to correctly identify patients has the potential to cause harm, e.g. when a patient with renal impairment and hyperkalemia receives the nutrition support meant for a patient in need of standard, highprotein nutrition support.

\section{Accuracy during care transitions}

Although accurate data on nutrition care during transitions are lacking, anecdotal evidence suggests that when patients move from one care area to another, problems commonly arise. The handover between care teams might not contain the essential nutrition care information, or the information might be misunderstood. These gaps in communication can cause a serious breakdown in continuity of care, inappropriate treatment and potential harm to patients. ${ }^{10}$ The Agency for Healthcare Research and Quality in the USA stated that according to the Joint Commission, approximately $70 \%$ of sentinel events in 2005 were caused by poor communication, and that half of those events occurred during patient handovers. ${ }^{11}$ Lessons on how to improve handovers are being learnt from other high-risk industries, such the aviation and nuclear power industries. One such lesson is the need for a common language when communicating critical information. Incorporating situational briefing techniques, such as the situation, background, assessment and recommendation (SBAR) process can provide a standard communication framework for patient care handover. The following example demonstrates how SBAR could be used to improve communication between two caregivers of a patient on nutrition support, in this case a dietitian, Ms A, and a physician, Dr M.

\section{Case study}

Ms $B$ is a critically ill patient on enteral nutrition support with a semielemental formula, formula X. She has developed severe diarrhoea despite the semi-elemental formula and other appropriate measures to manage the diarrhoea. During her daily assessment of the patient, Ms A notices in a new report from the laboratory that the patient was found to be Clostridium difficle-positive. The physician has not been performed his rounds yet, and it is unclear when he will see the patient. Ms A decides that to optimally feed the patient, total parenteral nutrition (TPN) is required. To prevent any delay, she calls Dr $\mathrm{M}$ to discuss the recommendation with him, and uses SBAR to communicate with him.
Situation: "Dr M, this is Ms A, the dietitian. I am calling you in connection with your patient in the medical intensive care unit, Ms B, medical record number 1234, with severe diarrhoea.

Background: "Despite being on enteral nutrition support with the appropriate semi-elemental formula $X$, her severe diarrhoea has not improved. I have noticed in a laboratory report dated today that she was found to be $C$. difficile-positive.

Assessment: "Optimal nutrition support via enteral feeding is not currently possible. The patient is at high risk of malnutrition.

Recommendation: "I recommend that we change the route of nutrition support to parenteral, and suggest that we start her on TPN formula P. If you agree, please see the patient urgently and prescribe the TPN formula $P$ to limit any delays in continued nutrition support. It would also be appreciated if you could request serum urea, creatinine, electrolytes, calcium, magnesium and phosphate tests for tomorrow morning to monitor Ms B's response to the TPN".

\section{Avoiding catheter and tubing misconnections}

Tubing, catheters and syringes are commonplace in health care, and are used to deliver medication and fluid to patients. Unfortunately, until recently, the design of these devices made it possible to inadvertently connect the incorrect tubing and syringes, and to deliver medication or fluid, including enteral and parenteral nutrition fluid, through the incorrect, unintended route. Other contributing factors included Luer connectors, the routine use of tubes or catheters for unintended purposes, positioning of functionally dissimilar tubes used in patient care in close proximity to one another, the movement of the patient from one setting or service to another and staff fatigue. ${ }^{12}$ The Joint Commission offers recommendations and strategies to healthcare organisations with regard to reducing tubing misconnection errors. Table I summarises these recommendations. ${ }^{13}$

Table I: Recommendations to reduce tubing misconnections

- Ensure that systems are in place which:

emphasize that only clinical staff may connect and disconnect devices

- require labeling of high risk catheters

- require that caregivers trace all lines from their origin to connection to verify attachments,

- include standardized line reconciliation process as part of handover communication

- bar the use of standard Luer-connection syringes to administer oral medications or enteric feedings.

- Incorporate training on the hazards of misconnecting tubing and devices into the orientation and continuing professional development of practitioners and health

- Promote the purchasing of tubes and catheters that are designed to enhance safety and to prevent misconnections with other devices or tubes

Many other solutions have been offered to solve this problem. Enteral tube connectors have been created in the enteral nutrition industry that are incompatible with other tube systems, although this has not yet been made mandatory. Tools have also been developed to improve the safety of enteral nutrition at the bedside. In an effort to improve enteral safety and to translate the enteral nutrition practice recommendations ${ }^{14}$ into bedside practice, in 2009, the American Society for Parenteral and Enteral Nutrition (ASPEN) launched a safety campaign aimed at educating nurses to help prevent enteral 
misconnections and to improve enteral nutrition safety. This initiative was called Be ALERT, and was followed by Be AWARE in 2010 and Be READY in 2011. The campaigns were sponsored by Nestlé HealthCare Nutrition. The mnemonics are represented on posters and remind caregivers of key practices that should be followed. The last one is specifically aimed at practising safe enteral feeding in children. ${ }^{15}$

Be ALERT is a mnemonic for:

- Aseptic technique: To be used in all enteral formula preparations and set up.

- Label the enteral equipment: To clearly identify the patient, clarify formula name and rate, date when started and identify the person responsible for starting the administration.

- Elevate the head of the bed: The head of the bed should be elevated when clinically possible. This may prevent the reflux of gastric content.

- Right patient, right formula and right tube: Match the formula and rate with the patient's feeding order and verify that the enteral tubing set connects the formula container to a feeding tube.

- Trace all lines back to the patient: Assure only enteral-to-enteral connections.

Be AWARE is a mnemonic for:

- Ask the pharmacist about drug-related matters: This includes drug-nutrient interactions, the correct dosage and safe drug administration via the feeding tube.

- Water only: Only water should be used to dilute medications and for the routine flushing of tubes, before and after medication administration.

- Administer the medication: Medication must be correctly administered, e.g. one medication at a time, and drugs identified that must be separated from the formula for more than 30 minutes.

- Remember the rights: This refers to the right patient, using the right drug, at the right dose, right route and right syringe.

- Establish evidence-based protocols: The institution's medication protocols must be followed and specific enteral medication protocols established.

Be READY is a mnemonic for:

- Right patient, right product and right tube: This must be followed to help to avoid misconnections and to ensure that human breast milk is labelled according to hospital policy.

- Early start to enteral feeding: It is important to start enteral feeding within 24-48 hours in a heamodinamically stable patient. Bowel sounds are not required for feeding to commence.

- Assure proper preparation and handling: Guidelines for the proper handling, preparation and storage of infant formula must be followed.

- Deliver the formula appropriately: This includes addressing the advancing of feeds, preventing interruptions, and ensuring correct hang times according to administration system and fluid status.

- Your safety checklist: It is important to check the placement of the enteral to enteral connections, that the correct syringes are used for the administration of medications through the feeding tube, to consult the paediatric pharmacist for any medications co-administered with the enteral feeds, and that the correct equipment is used to prevent tubing misconnections.

\section{Malnutrition and patient safety}

Malnutrition (undernutrition) is the result of an inadequate intake of protein and/or energy over time, resulting in the loss of fat and muscle stores. Inflammation may be an element of malnutrition. Malnutrition can be caused by starvation and acute and chronic illnesses that result in adverse effects on bodily functions and clinical outcome. This may occur with any body mass index. Table II describes the clinical characteristics of malnutrition, as described in a consensus statement by ASPEN and the Academy of Nutrition and Dietetics. ${ }^{16}$

Table II: The clinical characteristics of malnutrition

- Food intake less than the nutrition requirements

- Weight loss over time

- The loss of lean body mass

- The loss of fat mass

- Fluid accumulation

- Measurably diminished hand grip strength

It is estimated that one in three patients who is admitted to hospital is already malnourished. This is evident over the spectrum of patient care. Malnourished patients are two times more likely to develop a pressure an ulcer in hospital. ${ }^{17}$ On average, malnourished patients are hospitalised two days longer than those who are screened and treated early. ${ }^{18,19}$ Forty-five per cent of patients who fall down in hospital are malnourished. ${ }^{20}$ Patients with malnutrition or weight loss have three times the risk of acquiring surgical site infection. ${ }^{21}$

Studies have shown that nutrition intervention can significantly improve the outcomes of malnourished patients. There is a $25 \%$ reduction in pressure ulcer incidence, ${ }^{22}$ a $28 \%$ decrease in avoidable readmission, ${ }^{23} 14 \%$ fewer overall complications ${ }^{24}$ and the average length of stay is reduced by approximately two days. ${ }^{18,19}$ Therefore, it is clear that malnutrition can cause nutrition-related patient safety incidents.

\section{What is known about the safety culture of patients in South Africa?}

Data on patient safety in South African hospitals are lacking. There is an urgent need for data collection in order to obtain an overview of patient safety and nutrition care in hospitals and other care facilities. To achieve this, organisations such as the South African Society of Enteral and Parenteral Nutrition are encouraging healthcare institutions to participate in established initiatives to collect data, e.g. nutritionDay. nutritionDay is an international project, with a coordinating centre in Austria. Its aim is to improve knowledge and awareness of malnutrition by using a simple screening test for nutritional risk. It is a one-day cross-sectional audit, with an outcome evaluation for hospitals, intensive care units and nursing care homes. ${ }^{25}$

\section{The way forward}

There is a need to raise awareness of all issues relating to patient safety in South Africa if patient safety is to take its rightful place in the country's healthcare system. There is also a need to report on and analyse medical errors to be able to identify root causes behind the lack of patient safety and to address them. Although many 
institutions and care organisations collect data on an individual basis, there is a lack of combined data to inform patient safety in the South African healthcare system. Pronovost et al published a framework for patient safety research and improvement that could be used to organise patient safety research and improvement efforts. ${ }^{26}$ The local healthcare industry could learn from the Alliance to Advance Patient Nutrition. To raise awareness of the hidden costs of malnutrition and to promote the importance of nutrition in patients, a collaboration including ASPEN and the Academy of Nutrition and Dietetics was established in 2013. ${ }^{27}$ Better nutrition has a proven, positive impact on the quality of patient care and safety, and supports patients throughout all aspects of their treatment and recovery.

\section{Conclusion}

It is clear that there is a connection between nutrition and patient safety as it has been demonstrated that nutrition care has the potential to cause harm to patients. Malnutrition is linked to safe nutrition care and patient safety. With a growing population of older adults and an increase in chronic diseases, proper and safe nutrition care is even more important now than ever. ${ }^{28}$ However, a culture of patient safety must be established in the healthcare environment for safe nutrition care to be practised. This requires commitment at all levels from healthcare professionals. Dietitians, as experts in clinical nutrition, should be at the forefront of creating an institutional culture whereby stakeholders value nutrition. ${ }^{29}$ Only then will the true link between nutrition and patient safety be recognised, and given its rightful place in the care of patients.

\section{References}

1. Suñol R. Avedis Donabedian. Int J Qual Health Care. 2000;12(6):451-454

2. Kohn LT, Corrigan JM, Donaldson MS, editors, Committee on Quality of Health Care in America, Institute of Medicine. To err is human: building a safer healthcare system. Nationa Academies Press [homepage on the Internet]. 2000. Available from: http://www.nap.edu/ catalog/9728.html

3. Stelfox HT, Palmisani S, Scurlock C, et al. The "To err is human" report and patient safety literature. Qual Saf Health Care. 2006;15(3):174-178.

4. Richardson WE, Committee on Quality of Health Care in America, Institute of Medicine. Crossing the quality chasm: a new health system for the $21^{\text {st }}$ century. National Academies Press [homepage on the Internet]. 2001. c2013. Available from: http://www.nap.edu/ openbook.php?record id =10027\&page $=R 5$

5. Joint Commission International. Patient safety solutions preamble - May 2007. Patient safety solution 1. 2007. c2013. JCl [homepage on the Internet]. Available from: http://www. jointcommissioninternational.org/assets/3/7/PreambleandSolutionsENGLISH.pdf

6. McDowell MS. Clinical nutrition quality indicators and patient outcomes. Support Line. 2007;29(4):3-9.

7. Holmes B, Spiro A, Thomas JE, et al. Nutrition-related patient safety incidents. Proceedings of the Nutrition Society. 2010;69(OCE7):E529.

8. The Institute for Safe Medication Practices Canada. ALERT: Reports of severe harm after intravenous administration of breast milk to infants. ISMP Canada Safety Bulletin 2011:11(5):1-3 [homepage on the Internet]. c2013. Available from:

9. http://www.ismp-canada.org/download/safetyBulletins/ISMPCSB2011-05-ALERT-Reports of_Severe_harm_after_IV_admin_breast_milk.pdf

10. Joint Commission International. Patient safety solutions preamble - May 2007. Patient safety solution 2. c2013. Available from: http://www.jointcommissioninternational.org/assets/3/7/ PreambleandSolutionsENGLISH.pdf

11. Joint Commission International. Patient safety solutions preamble - May 2007. Patient safety solution 3. c2013. Available from: http://www.jointcommissioninternational.org/assets/3/7/ PreambleandSolutionsENGLISH.pdf

12. Agency for Healthcare Research and Quality. Transition "tickets" reduce adverse events during patient transports. AHRQ [homepage on the Internet]. 2013. Available from: http:// www.innovations.ahrq.gov/content.aspx?id=2313

13. Joint Commission International. Patient safety solutions preamble - May 2007. Patient safety solution 7. c2013. Available from: http://www.jointcommissioninternational.org/assets/3/7/ PreambleandSolutionsENGLISH.pdf

14. Joint Commission on Accreditation of Healthcare Organizations, USA. Tubing misconnections: a persistent and potentially deadly occurrence. Sentinel Event Alert. 2006;(36):1-3

15. Bankhead R, Boullata J, Brantley S, et al. Enteral nutrition practice recommendations. JPEN J Parenter Enteral Nutr. 2009;33(2):122-167.

16. American Society for Parenteral and Enteral Nutrition. A.S.P.E.N patient safety initiatives. ASPEN [homepage on the Internet]. c2013. Available from: https://www. nutritioncare.org/Guidelines_and_Clinical_Practice/Toolkits/Enteral_Nutrition_Toolkit/ Related_Publications_and_Tools/

17. White JV, Guenter P, Jensen G, et al. Consensus statement of the Academy of Nutrition and Dietetics/American Society for Parenteral and Enteral Nutrition: characteristics recommended for the identification and documentation of adult malnutrition (undernutrition). J Acad Nutr Diet. 2012;112(5):730-738.

18. Banks M, Bauer J, Graves N, Ash S. Malnutrition and pressure ulcer risk in adults in Australian health care facilities. Nutrition. 2010;26(9):896-901.

19. Brugler L, DiPrinzio MJ, Bernstein L. The five-year evolution of a malnutrition treatment program in a community hospital. Jt Comm J Qual Improv. 1999;25(4):191-206.

20. Smith $P E$, Smith $A E$. High-quality nutritional interventions reduce costs. Healthc Financ Manage. 1997:51(8):66-69

21. Bauer JD, Isenring E, Torma J, et al. Nutritional status of patients who have fallen in an acute care setting. J Hum Nutr Diet. 2007;20(6):558-564

22. Fry DE, Pine M, Jones BL, Meimban RJ. Patient characteristics and occurrence of never events. Arch Surg. 2010;145(2):148-151.

23. Stratton RJ, Elia M. Are oral nutritional supplements of benefit to patients in the community? Findings from a systematic review. Curr Opin Clin Nutr Metab Care. 2000;3(4):311-315.

24. Gariballa S, Forster S, Walters S, Powers H. A randomized, double-blind, placebo-controlled trial of nutritional supplementation during acute illness. Am J Med. 2006;119(8):693-699.

25. Milne AC, Potter J, Vivanti A, Avenell A. Protein and energy supplementation in elderly people at risk from malnutrition. [Cochrane review]. In: The Cochrane Library, Issue 2, 2009. 0xford: Update Software.

26. Nutrition Day Worldwide [homepage on the Internet]. c2013. Available from: http://www. nutritionday.org/index.php?id=9

27. Pronovost PJ, Goeschel CA, Marsteller JA, et al. Framework for patient safety research and improvement. Circulation. 2009;119(2):330-337.

28. The Alliance to Advance Patient Nutrition. Who we are [homepage on the Internet]. c2013. Available from: http://malnutrition.com/alliance

29. The Alliance to Advance Patient Nutrition. Why the time is now [homepage on the Internet] c2013. Available from: http://malnutrition.com/getinformed

30. The Alliance to Advance Patient Nutrition. Alliance nutrition care model and toolkit [homepage on the Internet]. c2013. Available from: http://malnutrition.com/getinvolved/ hospitalnutritiontoolkit 